www.elsevier.com/locate/nimb

\title{
Defect creation caused by the decay of cation excitons and hot electron-hole recombination in wide-gap dielectrics
}

\author{
A. Lushchik *, Ch. Lushchik, M. Kirm, V. Nagirnyi, F. Savikhin, E. Vasil'chenko \\ Institute of Physics, University of Tartu, Riia 142, 51014 Tartu, Estonia
}

Available online 12 June 2006

\begin{abstract}
Insufficient radiation resistance of construction materials is the Achilles heel for thermonuclear energetics. In wide-gap dielectrics, Frenkel defects are created not only because of the knock-out (impact) mechanism but also because of the decay of the electronic excitations formed during the irradiation (i.e. due to nonimpact mechanisms). The processes of the defect creation at the irradiation of highly pure LiF single crystals at $6-8 \mathrm{~K}$ by $1-30-\mathrm{keV}$ electrons, X-rays, or synchrotron radiation of $12-70 \mathrm{eV}$ have been investigated. The annealing processes of these defects in a temperature range up to $200 \mathrm{~K}$ have been studied as well. In LiF, creation has been revealed for the following: (1) $\mathrm{F}-\mathrm{H}$ pairs caused by the decay of anion excitons or by the recombination of electrons and holes, (2) $\mathrm{F}^{\prime}-\mathrm{H}-\mathrm{V}_{\mathrm{K}}$ and $\mathrm{F}-\mathrm{I}-\mathrm{V}_{\mathrm{K}}$ defect groups at the decay of cation excitons $(62 \mathrm{eV})$, or (3) $20-\mathrm{keV}$ electron irradiation. The mechanism of defect creation at the recombination of hot holes and hot electrons has been discussed for $\alpha-\mathrm{SiO}_{2}$ crystals with an energy gap between the subbands of a valence band. One of the possible ways to suppress this mechanism ("luminescent defence") is doping a material by luminescent impurities able to capture a part of the energy of hot carriers before their relaxation and recombination (e.g. in $\mathrm{MgO}: \mathrm{Cr}$ ).
\end{abstract}

(c) 2006 Elsevier B.V. All rights reserved.

PACS: $61.82 . \mathrm{Ms} ; 71.35 .-\mathrm{y} ; 78.60 .-\mathrm{Kn}$

Keywords: Hot recombination; Radiation defects; Cation excitons; Luminescence

\section{Introduction}

The awakened interest toward the urgent and extremely complicated problem of the increase of the radiation resistance of various construction materials (in particular, widegap dielectrics [1]) is connected with the recent efforts put forth to the development of deuterium-tritium fusion reactors for thermonuclear energetics. Thus, the reduction of defect creation by irradiation of wide-gap crystals (WGCs) is an important technical problem. Some of the consequences of solving this problem would be improved radiation resistance of windows for short-wavelength lithography and an increased lifetime of spectral transformers for plasma display panels. Several generations of scientists have devoted their efforts to the radiation resis-

\footnotetext{
* Corresponding author. Tel.: +372 738 3026; fax: +372 7383033.

E-mail address: luch@fi.tartu.ee (A. Lushchik).
}

tance of materials and any progress in this field is based on detailed investigations of the processes of defect creation in WGCs, especially in metal oxides and fluorides $\left(\mathrm{Al}_{2} \mathrm{O}_{3}, \mathrm{MgO}, \mathrm{SiO}_{2}, \mathrm{CaF}_{2}, \mathrm{LiF}\right.$, etc.).

It has long been established that the radiation defects in WGCs are created because of the knock-out (impact) mechanisms, universal for solids that are connected with elastic collisions of high-energy particles with the atoms/ ions of a crystal. The impact mechanisms have been studied in detail and the threshold particle energies for the creation of Frenkel defects (FDs) have been determined for many metal oxides (see [2] and references therein). It is commonly accepted that in WGCs (besides impact mechanisms of defect creation), the nonimpact mechanisms connected with the excitation and ionization of an electronic system by particles and quanta and the subsequent decay of various electronic excitations (EEs) into FDs should be considered (see, e.g. [3-6]). The efficiency of EE decay into FDs is 
especially high in WGCs where the creation energy of a pair of FDs $\left(E_{\mathrm{d}}\right)$ is lower than the value of the energy gap $\left(E_{\mathrm{d}}<E_{\mathrm{g}}\right)$ and the residence time of this EE on a certain lattice site is longer than the period of effective lattice vibrations. In such WGCs (e.g. in the majority of alkali halides), the efficiency of defect creation by the impact mechanism is significantly lower than at the decay of excitons or at the recombination of relaxed carriers. At the same time, according to theoretical calculations $[7,8]$ an inequality $E_{\mathrm{d}}>E_{\mathrm{g}}$ is valid in a number of WGCs. It was commonly supposed that the impact mechanisms of FD creation dominate in these materials, while the role of EEs in radiation damage is negligible [4-6]. This energy criterion applies to the lowest-energy EEs (usually anion excitons). However, in addition to these EEs, short-lived EEs (e.g. cation excitions [4,9]) are created whose energies exceed the defect creation energy $E_{\mathrm{d}}$. Thus, these EEs may create defects. Furthermore, in addition to the recombination of relaxed (cold) carriers, the recombination of hot (nonrelaxed) electrons and hot holes is possible as well (see, e.g. [10]).

The aim of the present study is to consider new experimental data on the role of cation excitons and the recombination of hot electrons with hot holes in the defect creation in some WGCs based on metal oxides and fluorides with various values of $E_{\mathrm{g}}$, formation energies of anion and cation excitons ( $E_{\mathrm{ea}}$ and $E_{\mathrm{ec}}$ ) and the width of a valence band $\left(E_{\mathrm{v}}\right)$.

\section{Decay of EEs with the defect creation in LiF and NaF crystals}

Let us first consider the peculiarities of the radiative and nonradiative decay of intrinsic anion and cation EEs in LiF crystals which are widely used in personal dosimetry and short-wavelength optics. Band structure calculations, which consider electron-hole (e-h) interaction, have been performed for $\mathrm{LiF}[11,12]$. The optical characteristics of LiF have been experimentally studied in spectral regions up to $40 \mathrm{eV}$ [13] and $1000 \mathrm{eV}$ [14]. Recently, we conducted a comparative investigation of optical characteristics in several LiF crystals that have a different purity level and are grown by various methods $[15,16]$. This paper discusses the new experimental results obtained for the purest $\mathrm{LiF}$ samples (named as LiF-1 in our previous studies) using synchrotron radiation (SUPERLUMI and BW3 stations of HASYLAB at DESY, Hamburg and beamline BL52 at MAX-I Laboratory, Lund) and the sample irradiation by an electron beam $(1-30 \mathrm{keV}, 10-100 \mathrm{nA}$, beam spot $\sim 0.5 \mathrm{~mm}^{2}$ ). The experimental details are described in $[15,16]$. The LiF crystals were grown by the Stockbarger method after a purification cycle involving manyfold zone-melting.

Fig. 1 presents the optical characteristics of LiF measured in a long-wavelength region of fundamental absorption at $9 \mathrm{~K}$. The reflection maximum at $12.96 \mathrm{eV}$ and a minimum at $13.83 \mathrm{eV}$ correspond to the creation of transverse and longitudinal anion excitons, respectively. The

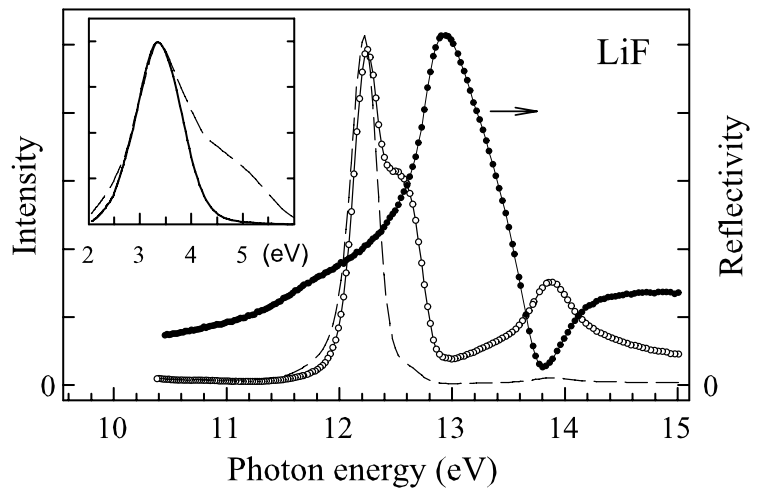

Fig. 1. The reflection spectrum (O) and the excitation spectra of 3.4-eV (OO) and 4.2-eV emissions (dashed line) measured for a LiF crystal at $9 \mathrm{~K}$. The inset shows the emission spectra measured at the excitation of a LiF crystal by $13-\mathrm{eV}$ (solid line) or $16.2-\mathrm{eV}$ photons (dashed line) at $9 \mathrm{~K}$.

energy gap $E_{\mathrm{g}} \cong 14.2 \mathrm{eV}$ [16]. The broad emission band, peaked at $3.4 \mathrm{eV}$ and ascribed to the radiative decay of triplet self-trapped excitons (STEs), is excited by $13-\mathrm{eV}$ photons, which directly form anion excitons, and also by 16.2-eV photons generating separated electrons and holes. In addition, the 4.7-eV impurity emission is also excited by 16.2-eV photons (see inset in Fig. 1). The excitation spectrum of the STE emission, normalized to equal quantum intensities of synchrotron radiation, spans the energy region of exciting photons of $h v_{\mathrm{exc}} \geqslant 11.8 \mathrm{eV}$. However, a narrow excitation band at $12.2 \mathrm{eV}$ can be also detected in the excitation spectrum for $4.2-\mathrm{eV}$ emission. According to theoretical estimations, the $12.2 \mathrm{-eV}$ band corresponds to the absorption of surface excitons in $\mathrm{LiF}$ [12]. The 4.2-eV emission is not excited at $h v_{\mathrm{exc}}<11.5 \mathrm{eV}$ and in the whole region of fundamental bulk absorption, that is not typical of impurity emissions in LiF.

Fig. 2 shows the curves of thermally stimulated luminescence (TSL) measured with a constant heating rate of $\beta=10 \mathrm{~K} \mathrm{~min}^{-1}$ for $\mathrm{LiF}$ and LiF:Na ( $\geqslant 100 \mathrm{ppm}$ ) crystals irradiated by an electron beam at $6 \mathrm{~K}$. In a highly pure

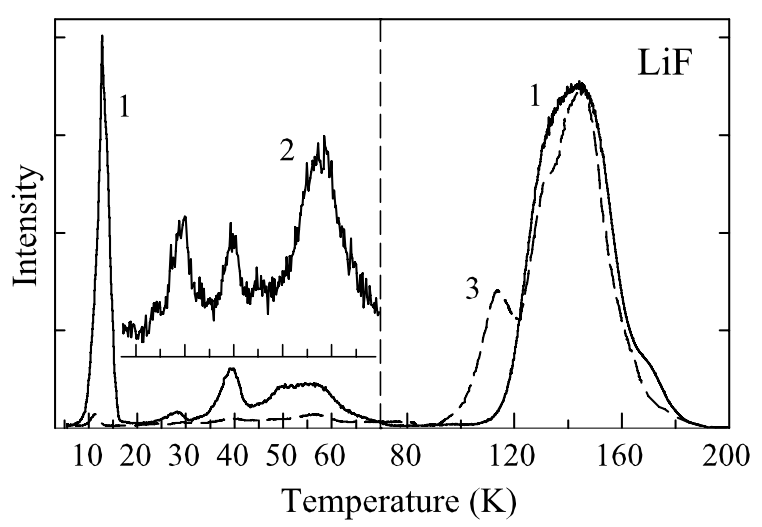

Fig. 2. The TSL curves measured for 3.4-eV (curve 1) and 4.6-eV emission (curve 2) in $\mathrm{LiF}$ and for the 2.7-eV emission (curve 3, dashed) in $\mathrm{LiF}: \mathrm{Na}$ crystals irradiated by an electron beam $\left(30 \mathrm{keV}, 10 \mathrm{nA} \mathrm{mm}^{-2}\right)$ at $6 \mathrm{~K}$, $\beta=10 \mathrm{~K} \mathrm{~min}^{-1}$. 
LiF crystal, the TSL curve contains an intense narrow peak at $13 \mathrm{~K}$, several peaks at $20-70 \mathrm{~K}$, the relative intensities of which depend on the emissions selected through a double prism monochromator (the 3.4-eV emission of STEs and 4.6-eV impurity emission) and the peaks at $120-180 \mathrm{~K}$ usually ascribed to a hopping diffusion of self-trapped holes, the so-called $V_{K}$ centers. According to Fig. 2, the presence of $\mathrm{Na}^{+}$impurity ions significantly suppresses the $13 \mathrm{~K} \mathrm{TSL}$ peak, decreases the intensity of TSL at $20-70 \mathrm{~K}$ by several times, and causes a sharp increase of the TSL peak at $113 \mathrm{~K}$.

Fig. 3 presents the pulse-annealing curve of the EPR signal of $\mathrm{V}_{\mathrm{K}}$ centers for LiF X-irradiated at $77 \mathrm{~K}$ [16] and the curves of integral TSL for a sample previously irradiated at $8 \mathrm{~K}$ by $13.8-$ and $62-\mathrm{eV}$ photons, which selectively form anion and cation excitons, respectively. A narrow absorption band at $62 \mathrm{eV}$ [14] and the approximate resonance to its X-emission [17] in the region of $1 s^{2} \leftrightarrow 1 \mathrm{~s} 2 \mathrm{p}$ transitions in $\mathrm{Li}^{+}$testify to the existence of cation excitons in $\mathrm{LiF}$. Selective creation of anion excitons in $\mathrm{LiF}$ causes the appearance of the $132 \mathrm{~K}$ TSL peak connected with a hopping diffusion of $\mathrm{V}_{\mathrm{K}}$ centers as well as the peak at $113 \mathrm{~K}$ whose intensity sharply increases in LiF that contain impurity sodium ions. $\mathrm{H}_{\mathrm{A}}\left(\mathrm{Na}^{+}\right.$) centers (an $\mathrm{H}$ center localized near a $\mathrm{Na}^{+}$impurity ion) with the orientation along $\langle 110\rangle$ and the annealing at $110-120 \mathrm{~K}$ have been detected in X-irradiated LiF:Na by means of ENDOR method [18]. At the same time, intrinsic $\mathrm{H}$ centers (anion interstitial atoms) oriented along $\langle 111\rangle$ in a regular lattice region and stable up to $60 \mathrm{~K}$ have been also detected in LiF using the EPR method [19]. The TSL peak at $\sim 65 \mathrm{~K}$ corresponding to the annealing of $\mathrm{H}$ centers arises in a $\mathrm{LiF}$ sample previously irradiated by $62-\mathrm{eV}$ photons (see Fig. 3). According to [16], the thermal ionization and the annealing of $\mathrm{F}^{\prime}$ centers (two electrons in a field of an anion vacancy) take place at $150-170 \mathrm{~K}$. Thus, the efficient creation of $\mathrm{F}^{\prime}$ centers occurs in a $62-\mathrm{eV}$ photon irradiated LiF due to the decay of cation excitons (see the corresponding TSL peak in Fig. 3).

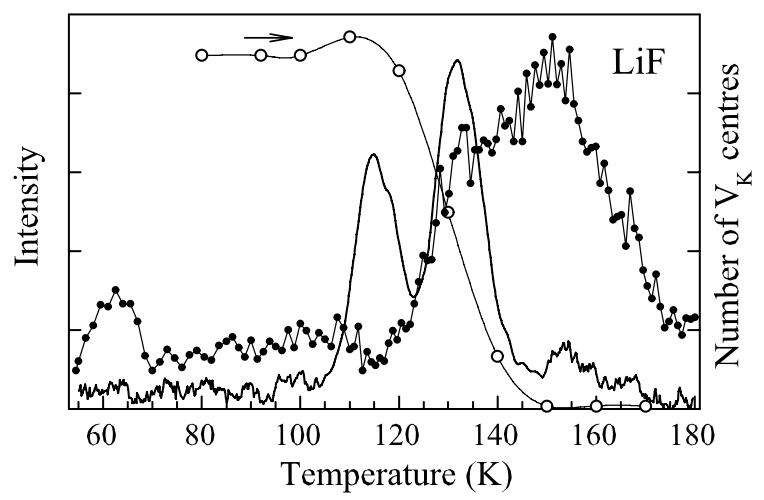

Fig. 3. The TSL curves measured for an integral signal in a LiF crystal irradiated by $13.8-\mathrm{eV}$ (solid line) or $62-\mathrm{eV}(\bullet \bullet)$ photons at $8 \mathrm{~K}$. The pulse annealing of the EPR signal of $\mathrm{V}_{K}$ centers $(\mathrm{O})$ in a crystal X-irradiated $(50 \mathrm{keV})$ at $77 \mathrm{~K}[16]$.
Of particular interest is the creation mechanism of TSL at $10-15 \mathrm{~K}$. This TSL peak can be easily detected for 2.9-, 3.4- and 5.7-eV emissions in relatively pure LiF samples preliminarily irradiated by $1.5-30 \mathrm{keV}$ electrons or X-rays $(20-50 \mathrm{keV})$ at $4-9 \mathrm{~K}$. However, the lowest-temperature TSL peak has not been detected after LiF irradiation, selectively forming at $8 \mathrm{~K}$ anion excitons (12.5- or $13.8-\mathrm{eV}$ photons) or separated electrons and holes (photons of 15 and $17 \mathrm{eV}$ ). It was proposed that the $13 \mathrm{~K}$ TSL peak arises only under the conditions of a high local density of the EEs formed at the irradiation [16]. Such irradiation conditions are realized at the multiplication of EEs (the photons of $h v_{\mathrm{exc}}=28-32 \mathrm{eV}$ are needed for this process [16]) or at the decay of cation excitons $\left(h v_{\mathrm{exc}}=62 \mathrm{eV}\right)$. According to our latest data, the irradiation of LiF by $62 \mathrm{eV}$ photons causes the appearance of the TSL at $50-180 \mathrm{~K}$ (see Fig. 3) and the peak in the region of $10-15 \mathrm{~K}$ whose intensity is about three times as high as that for the $60 \mathrm{~K}$ peak.

The decay of a cation exciton, formed by a $33-34-\mathrm{eV}$ photon in a $\mathrm{NaCl}$ crystal, into an anion exciton and two electron-hole $(\mathrm{e}-\mathrm{h})$ pairs has been earlier revealed using highly sensitive luminescent methods [9,20]. Agranovich and Ginzburg [21] theoretically considered a short-wavelength analogue of the Raman effect, when excitons act as phonons at the scattering of X-rays. We used this idea as a starting point for the hypothesis for the decay of a cation exciton with the creation of an anion exciton. In LiF, a photon of $h v_{\mathrm{exc}}=E_{\mathrm{ec}}=62 \mathrm{eV}$ can be transformed into an anion exciton $\left(E_{\mathrm{ea}} \approx 13 \mathrm{eV}\right)$, while the rest of the energy $(\approx 49 \mathrm{eV})$, after intermediate processes, is expended by creating up to three $\mathrm{e}-\mathrm{h}$ pairs. As this takes place, an anion exciton and at least one of the holes undergo rapid selftrapping spatially close to each other. Thereafter, similar to other alkali halides [3-6], an anion STE decays into an $\mathrm{F}-\mathrm{H}$ pair of FDs. As a result, a triplet of $\mathrm{F}-\mathrm{H}-\mathrm{V}_{\mathrm{K}}$ defects is formed. One of the conduction electrons participates in a subsequent recharging of $\mathrm{F}$ into $\mathrm{F}^{\prime}$ or $\mathrm{H}$ into an I center (an interstitial anion) forming $\mathrm{F}^{\prime}-\mathrm{H}-\mathrm{V}_{\mathrm{K}}$ or $\mathrm{F}-\mathrm{I}-\mathrm{V}_{\mathrm{K}}$ groups of spatially correlated defects.

The tunnelling of either of the two electrons from an $\mathrm{F}^{\prime}$ center to a $\mathrm{V}_{\mathrm{K}}$ center in an $\mathrm{F}^{\prime}-\mathrm{H}-\mathrm{V}_{\mathrm{K}}$ triplet in $\mathrm{LiF}$ causes both (i) a direct radiative transition into a ground state of a $\mathrm{V}_{\mathrm{K}}$ center ( $\sim 5.7 \mathrm{eV}$ emission) or (ii) the creation of an STE with the subsequent emission of a 3.4-eV photon. Within $\mathrm{F}-\mathrm{I}-\mathrm{V}_{\mathrm{K}}$ triplets in $\mathrm{LiF}$, one would expect the appearance of the 3.4-eV emission at the tunnel recharging of $\mathrm{F}-\mathrm{V}_{\mathrm{K}}$ into an STE and an anion vacancy. Previously we have detected the creation of such defect groups at the irradiation of a $\mathrm{KBr}$ crystal by $16-\mathrm{eV}$ photons at $8 \mathrm{~K}$ [22], i.e. under the conditions of multiplication of EEs, when a hot primary conduction electron is able to form a secondary anion exciton [23], or at X-irradiation of $\mathrm{KCl}$ at $4 \mathrm{~K}$ [24]. It is significant that under the conditions of a high local density of EEs, triplets of both types stable at 6$8 \mathrm{~K}$ can be formed not only from long-lived $\mathrm{F}-\mathrm{H}$ pairs with interdefect separation in the pairs $r_{\mathrm{FH}}=4-5$ interanion distances, but also from short-lived $\left(10^{-3}-10^{2} \mathrm{~s}\right)$ close $\mathrm{F}-\mathrm{H}$ 
pairs. This circumstance explains highly efficient creation of the $13 \mathrm{~K}$ TSL peak in a LiF crystal, where the crowdion motion of $\mathrm{H}$ interstitials along $\langle 110\rangle$ anion rows is impeded and the values of $r_{\mathrm{FH}}$ are low.

Fig. 4 shows the TSL curves measured for 5.4- and 3.4-eV emissions at $7-16 \mathrm{~K}$ for an electron-irradiated $\mathrm{LiF}$ crystal at $6 \mathrm{~K}$. The curves have been quantitatively analysed by decomposing into the components described by first-order kinetics. The TSL of $5.4 \mathrm{eV}$ (tunnel transitions in $\mathrm{F}^{\prime}-\mathrm{V}_{\mathrm{K}}$ ) consists of a single peak with the maximum at $12.7 \mathrm{~K}$ with the activation energy of $\varepsilon=9.5 \mathrm{meV}$ and the frequency factor of $p=5.8 \times 10^{2} \mathrm{~s}^{-1}$ (see Fig. 4(a)). The TSL curves measured for $3.4-\mathrm{eV}$ emission contains two components: a broad peak characterized by approximately the same values of $\varepsilon$ and $p$ and a second peak with $\varepsilon \approx 25 \mathrm{meV}$ and $p \approx 10^{9} \mathrm{~s}^{-1}$ (Fig. 4(b)). Both TSL components can be sharply depressed by the additional illumination of the irradiated sample at $6 \mathrm{~K}$ by $2.4-2.8-\mathrm{eV}$ photons from the region of $\mathrm{F}^{\prime}$-absorption band. Such photostimulation also causes the appearance of the 3.4-eV optical flash. It should be mentioned that the values of $\varepsilon$ for both TSL components correspond to the energy region of acoustic lattice vibrations of $\mathrm{LiF}$ [16].

We made an attempt to investigate the peculiarities of high-temperature TSL in an NaF crystal irradiated by synchrotron radiation of 18 or $33 \mathrm{eV}$ at room temperature. The integral TSL was measured when the irradiated sample was heated with a constant rate of $\beta=2.86 \mathrm{~K} \mathrm{~s}^{-1}$ in an atmosphere of flowing nitrogen (System 310 TLD Reader). Fig. 5 demonstrates the TSL curves measured after equal quantum dose irradiation of $\mathrm{NaF}$ by $18 \mathrm{eV}$ photons, each creating a single $\mathrm{e}-\mathrm{h}$ pair, or by $33 \mathrm{eV}$ photons selectively forming cation excitons, which decay with the formation of an anion exciton $\left(E_{\mathrm{ea}}=10.8 \mathrm{eV}\right.$ at $\left.7 \mathrm{~K}\right)$ and one e-h pair $\left(E_{\mathrm{g}}=12.3 \mathrm{eV}\right)$ [25]. It is important to remember that the absorption and the reflection constants for 18 and $33 \mathrm{eV}$ photons have approximately the same values [13]. Nevertheless, the total light sum of TSL induced by a $33-\mathrm{eV}$

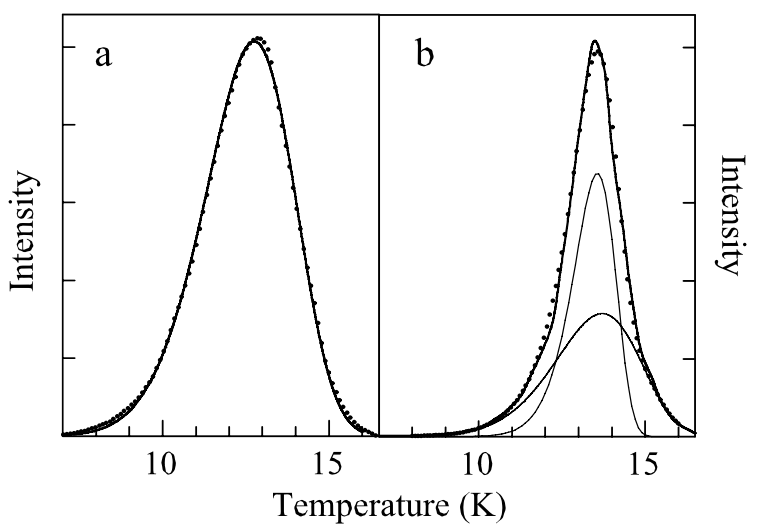

Fig. 4. The TSL curves measured for 5.4- (a) or 3.4-eV (b) emission in a $\mathrm{LiF}$ crystal irradiated by $30 \mathrm{keV}$ electrons at $6 \mathrm{~K}, \beta=10 \mathrm{~K} \mathrm{~min}^{-1}$. The experimental curve (O) and the decomposition components described by the first order kinetics (thin solid lines) and their sum (thick lines).

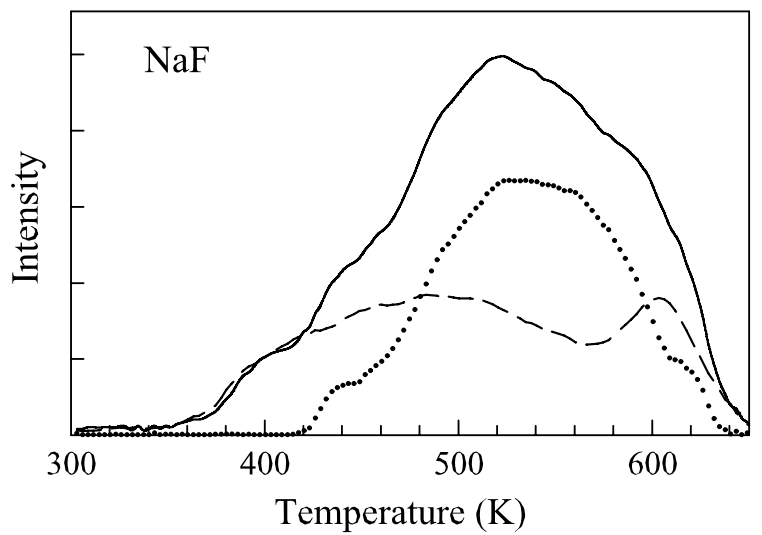

Fig. 5. The integral TSL measured for an NaF crystal irradiated by equal quantum doses of 18 (dashed line) and $33-\mathrm{eV}$ photons (solid line) at $295 \mathrm{~K}$. The curves were measured with a heating rate of $\beta=2.86 \mathrm{~K} \mathrm{~s}^{-1} 48 \mathrm{~h}$ after the irradiation was stopped. The difference between the curves for the 30 $\mathrm{eV}$ and $18-\mathrm{eV}$ irradiation (dotted line).

photon irradiation is more than three times as high as that after an $18-\mathrm{eV}$ photon irradiation. These results show that especially favorable conditions for the stabilization of radiation defects at $295 \mathrm{~K}$ in fluorides occur at a high local density of the EEs formed at the decay of cation excitations. We observed a similar effect earlier at the irradiation of a $\mathrm{KBr}$ crystal at $295 \mathrm{~K}$ by $16-\mathrm{eV}$ photons, the energy of which is sufficient for the creation of an $\mathrm{e}-\mathrm{h}$ pair and a secondary anion exciton [26].

\section{Hot $\mathrm{e}-\mathrm{h}$ recombination and defect creation in oxides}

Now we will look more closely at the role of hot electrons, hot holes, and their recombination in the creation of lattice defects. In our opinion, this problem is especially important for wide-gap metal oxides with wide and structured valence bands (v-bands). The radiation effects caused by recombination of hot carriers can be more easily separated in metal oxides where $E_{\mathrm{d}}>E_{\mathrm{g}}$. According to the investigations of fast relaxation processes of conduction electrons in WGCs (see, e.g. [27-29] and references therein), the efficient creation of a secondary e $-\mathrm{h}$ pair by a hot electron of a sufficient energy takes place in a subpicosecond timescale. However, if the energy of a hot electron is insufficient for the formation of a secondary intrinsic EE, the speed of its relaxation in regular lattice sites significantly slows down. In alkali halides with the content of impurity ions about $10^{3} \mathrm{ppm}$, such hot electrons can transfer their excess energy to luminescent impurity ions before their total energy relaxation [30].

The relaxation processes of hot holes in WGCs are poorly understood. Considering the width of valence bands and their complex structure, we can assume that the relaxation of hot holes with the formation of phonons takes place with time delays, thus, increasing the probability of the recombination of conduction electrons with hot holes. The presence of the energy gaps between the subbands of 


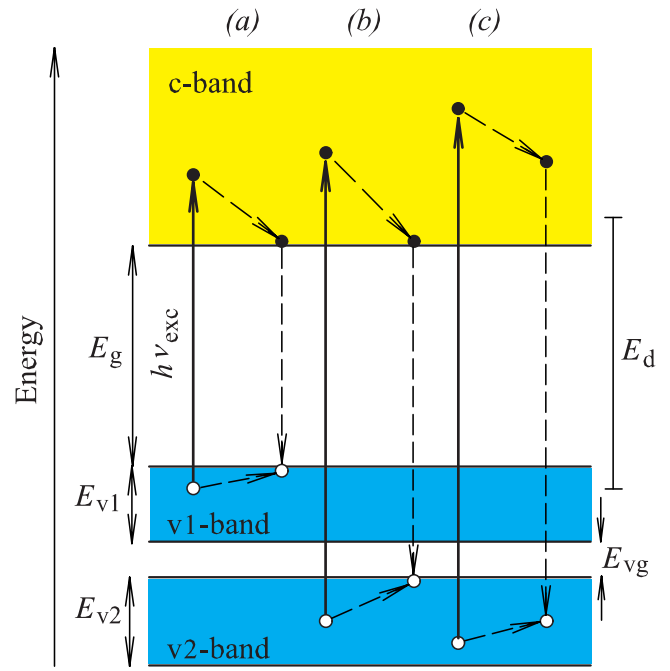

Fig. 6. A simplified energy-band diagram of a wide-gap dielectric with the creation energy of a pair of FDs higher than the value of the energy gap, $E_{\mathrm{d}}>E_{\mathrm{g}} . E_{\mathrm{v} 1}$ and $E_{\mathrm{v} 2}$ are the widths of the valence subbands separated by the energy gap $E_{\mathrm{vg}}$. All the three processes start with the absorption of an exciting photon (showed by a solid arrow line). The inclined dashed arrow lines demonstrate the vibrational relaxation of carriers inside the energy bands and the vertical ones - nonradiative transitions at the e- $h$ recombination without defect creation (a) or with a possible defect creation $(b, c)$.

a split v-band can drastically increase the probability of hot recombination between carriers in WGCs.

Fig. 6 presents a simplified energy band diagram of a WGC with two valence subbands (width of $E_{\mathrm{v} 1}$ and $E_{\mathrm{v} 2}$, respectively) separated by the energy gap $E_{\mathrm{vg}}$. In a crystal with a high energy of FD creation, $E_{\mathrm{d}}>E_{\mathrm{g}}$, the case (a) demonstrates fast relaxation of the hot electrons and hot holes, formed by the absorption of an exciting photon, to the bottom of the conduction band (c-band) and the top of the v-band, respectively. The energy released at the recombination of such totally relaxed carriers is insufficient for the creation of a pair of FDs. On the other hand, if the relaxation of hot holes occurs only up to the top of the lower subband of the v-band (case (b)), the energy released at a subsequent recombination equals $E=E_{\mathrm{g}}+E_{\mathrm{v} 1}+E_{\mathrm{vg}}$ and is sufficient for the defect creation $\left(E>E_{\mathrm{d}}\right)$. The case (c) is realized under the conditions of high-density excitation and the released energy can even reach the value of $2 E_{\mathrm{g}}$. If $h v_{\mathrm{exc}}>2 E_{\mathrm{g}}$, the process of the multiplication of EEs, i.e. the creation of secondary cold $\mathrm{e}-\mathrm{h}$ pairs, starts and the relaxation time of hot carriers sharply decreases. In some WGCs, the theoretically estimated value of $E_{\mathrm{d}}$ only slightly exceeds $E_{\mathrm{g}}$. Thus, in our opinion, hot recombination of carriers in such materials (especially with a wide and split v-band) can lead to the creation of FDs.

A weak fast intraband luminescence (IBL) connected with the radiative transitions of hot electrons between the levels of the c-band (the so-called e-IBL) was discovered in alkali halides [31,32]. In wide-gap metal oxides, the more intense h-IBL due to the transitions of hot holes between the levels of the v-band has also been revealed [33]. A

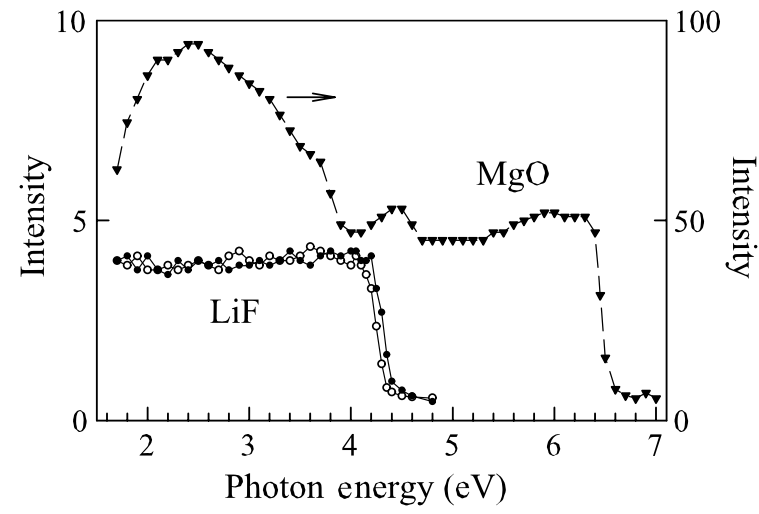

Fig. 7. The spectra of intraband luminescence $(\tau<2 \mathrm{~ns})$ measured for $\mathrm{LiF}$ at $80(\bigcirc \bigcirc)$ or $300 \mathrm{~K}(\bullet)$ and for a $\mathrm{MgO}$ crystal at $730 \mathrm{~K}(\boldsymbol{\nabla} \boldsymbol{\nabla})$ under irradiation by single $300 \mathrm{keV}$ electron pulses.

short-wavelength edge of the h-IBL allows an estimate of the total width of the band, $E_{\mathrm{v}}$ [33-35]. The value of $E_{\mathrm{v}}$ equals 8.7, 6.6 and $6.9 \mathrm{eV}$ in $\mathrm{BeO}, \mathrm{MgO}$ and $\mathrm{BaMgAl}_{10} \mathrm{O}_{17}$, respectively.

Fig. 7 shows new data on the h-IBL obtained at the irradiation of $\mathrm{LiF}$ and $\mathrm{MgO}$ by single electron pulses from the Koval'chuk-Mesyats type generator $\left(3 \mathrm{~ns}, 80 \mathrm{~A} \mathrm{~cm}^{-2}\right.$, $300 \mathrm{keV})$. In highly pure $\mathrm{LiF}$, there are no fast $(\tau<2 \mathrm{~ns})$ impurity emissions in a spectral region of up to $12 \mathrm{eV}$ even at 80 or $295 \mathrm{~K}$. However, a structureless emission at 1.6$4.3 \mathrm{eV}$ that results from the h-IBL can easily be detected in fresh LiF crystals or preliminarily X-irradiated samples. The intensity of the e-IBL in LiF is low because of fast relaxation of the electrons inside a wide c-band and therefore can be detected only at $h v>4.4 \mathrm{eV}$. Fig. 7 also presents the spectrum of the h-IBL ( $\tau<2 \mathrm{~ns}$ ) in an extremely pure $\mathrm{MgO}$ single crystal. A short-wavelength edge of the spectrum is located at $6.6 \mathrm{eV}$. At 80 or $295 \mathrm{~K}$, the IBL spectrum at $1.6-5.8 \mathrm{eV}$ is complicated because of the overlapping of several near-impurity emissions, the contribution of which can be practically eliminated by the sample heating to $730 \mathrm{~K}$. The general shape of the IBL spectrum agrees with the density of states and the width of the v-band in $\mathrm{MgO}$ calculated in several reports (see, e.g. [36]). Contrary to the case of $\mathrm{MgO}$, the energy gap between the valence subbands has been theoretically predicted [37] and experimentally detected by measuring the spectra of the hole IBL in some sulphates (e.g. in $\mathrm{K}_{2} \mathrm{SO}_{4}$ ) [34]. The first band structure calculation did not predict the energy gap between the valence subbands of $\alpha$-quartz [38]. However, the value of $E_{\mathrm{vg}} \approx 1.5 \mathrm{eV}$ was obtained in more advanced calculations (see, e.g. $[39,40]$ ).

Of particular interest is the role of hot recombination in defect creation in highly pure $\alpha-\mathrm{SiO}_{2}$ crystals ( $\alpha$-quartz). Efficient defect creation by irradiation of $\alpha$-quartz by 6$\mathrm{keV}$ electrons at $9 \mathrm{~K}$ has been detected by measuring the rise of the $5-\mathrm{eV}$ emission intensity (this emission is connected with radiation defects) during $3 \mathrm{~h}$ irradiation [41]. It was shown [42] that the same defects (absorption band at $7.6 \mathrm{eV}$ ) are formed under $\gamma$-irradiation with the density 
of excitation lower than that under the electron irradiation. A relatively high radiation resistance of pure $\alpha-\mathrm{SiO}_{2}$ was earlier explained by the fact that the inequality $E_{\mathrm{d}}>E_{\mathrm{g}}=8.9 \mathrm{eV}$ is valid in crystalline $\alpha$-quartz in contrast with amorphous silica. Therefore, it was difficult to expect the creation of FDs in a perfect lattice due to the recombination of relaxed (cold) electrons and holes. A new hypothesis has recently been proposed about a possible FD creation in $\alpha-\mathrm{SiO}_{2}$ at hot recombination of carriers, when the released energy can significantly exceed the value of $E_{\mathrm{g}}$ [10]. Also emphasized was the role of the enhanced excitation density in the creation of defects at the irradiation of $\alpha$-quartz by an electron beam at $9 \mathrm{~K}$ [41].

In $\alpha-\mathrm{Al}_{2} \mathrm{O}_{3}$ and aluminosilicate ceramics, the photoionization of the $2 \mathrm{~s}^{2}$ shell of oxygen ions causes the formation of secondary e-h pairs due to an Auger process with the participation of $2 \mathrm{~s}$ and $2 \mathrm{p}$ oxygen shells [43,44]. As a result, $\mathrm{e}-\mathrm{h}-\mathrm{h}$ groups of EEs are formed, thus providing especially favorable conditions for the creation of temperature-stable F centers in $\mathrm{Na}_{6} \mathrm{Al}_{6} \mathrm{Si}_{6} \mathrm{O}_{24}(\mathrm{NaBr})_{1.9}$ optical ceramics [44]. It is not inconceivable that a similar process can increase the efficiency of low-temperature defect creation in $\alpha-\mathrm{SiO}_{2}$, where $2 \mathrm{~s}^{2} \mathrm{O}$ shell ionisation is theoretically predicted at $h v_{\mathrm{exc}}=27-29 \mathrm{eV}[40]$.

\section{Possibilities of the suppression of hot $\mathrm{e}-\mathrm{h}$ recombination in dielectrics}

There are at least two possibilities to suppress the recombination of the hot electrons and hot holes in dielectrics with $E_{\mathrm{d}}>E_{\mathrm{g}}$. The energy gap between two valence subbands of $\alpha$-quartz can be eliminated by preliminary processing of the material (e.g. by fluorination or deformation of $\mathrm{SiO}_{2}$ ). The elimination of $E_{\mathrm{vg}}$ causes fast relaxation of the holes inside the v-band, thus decreasing the efficiency of hot $\mathrm{e}-\mathrm{h}$ recombination.

On the other hand, according to band-gap calculations, there is no energy gap inside a v-band of $\mathrm{MgO}$ and $\mathrm{Al}_{2} \mathrm{O}_{3}$. The doping of such crystals with certain luminescent impurities can reduce the efficiency of the recombination of hot carries. When the energy of hot conduction electrons, with respect to the bottom of the c-band, decreases approximately to (slightly below) $E_{\mathrm{g}}$, the recombination velocity of these electrons slows down because they are no longer able to create secondary e-h pairs (or secondary excitons). However, hot electrons efficiently interact with impurities and expend their excess energy for the excitation of luminescent impurity centers. As a result, the recombination of these electrons with holes does not lead to the creation of FDs $\left(E_{\mathrm{d}}>E_{\mathrm{g}}\right)$.

In alkali halide crystals doped with $\mathrm{Tl}^{+}$or $\mathrm{Ag}^{+}$impurity ions (up to 100-300 ppm), the energy of hot conduction electrons or hot holes is expended by the efficient excitation of impurity centers or halogen ions adjacent to impurity ions even at $8 \mathrm{~K}[30,45]$. More complicated process of the energy transfer by hot holes, formed by VUV radiation, to luminescent $\mathrm{Eu}^{2+}$ centers has been revealed in $\mathrm{BaMgAl}_{10} \mathrm{O}_{17}$ : Eu and

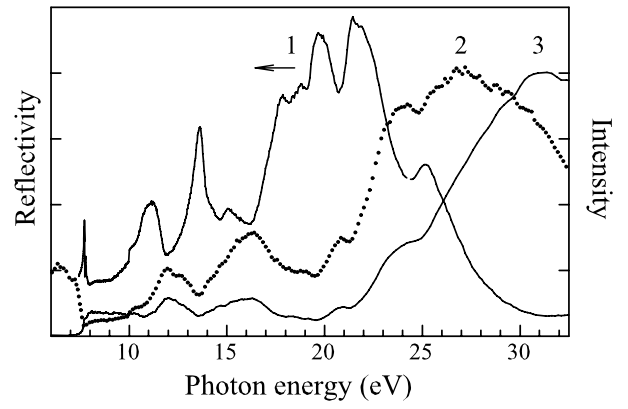

Fig. 8. The reflection spectrum (1) for a $\mathrm{MgO}$ crystal and the excitation spectra of the 1.7-eV emission (2, dotted line) in $\mathrm{MgO}: \mathrm{Cr}(60 \mathrm{ppm})$ and 5.4-eV emission (3) for MgO:Al, $T=8 \mathrm{~K}$.

$\mathrm{SrMgAl}_{10} \mathrm{O}_{17}: \mathrm{Eu}$ phosphors with wide v-bands, spectral transformers for plasma display panels [35]. The efficiency of this process is high if the concentration of europium ions exceeds $1000 \mathrm{ppm}$.

Of particular interest is the possibility of the reduction of the recombination efficiency of hot carriers in a row of $\mathrm{MgO}: \mathrm{Cr}^{3+}$ crystals, where the chromium concentration varies from 10 to $600 \mathrm{ppm}$. The peculiarities of EEs were earlier studied in highly pure and doped with $\mathrm{Al}^{3+}, \mathrm{Ca}^{2+}$ and $\mathrm{F}^{-}$single crystals of $\mathrm{MgO}$ using synchrotron radiation of $5-35 \mathrm{eV}$ [46]. We have continued these investigations. Fig. 8 presents the reflection spectrum measured at $8 \mathrm{~K}$ for the (100) plane of $\mathrm{MgO}$ after a crystal cleavage in situ under ultrahigh-vacuum conditions at $295 \mathrm{~K}$. A distinct structure at $7.68-7.75 \mathrm{eV}$ was interpreted as the formation of large-radius Wannier excitons. A broadband emission at $5.4 \mathrm{eV}$ in $\mathrm{MgO}: \mathrm{Al}$ corresponds to the recombination of electrons with the holes localized at the oxygen near the associations of cation vacancies with $\mathrm{Al}^{3+}$ impurity ions. The threshold energy of the excitation of this emission is close to the value of $E_{\mathrm{g}}=7.8 \mathrm{eV}$. A sharp rise of the 5.4-eV emission efficiency occurs at $22 \mathrm{eV}$, i.e. at $h v_{\mathrm{exc}}>2 E_{\mathrm{g}}+E_{\mathrm{v}}$ and continues up to $31 \mathrm{eV}$ due to the creation of secondary e $\mathrm{h}$ pairs by hot photoelectrons.

The excitation spectrum for the R-line $(1.7 \mathrm{eV})$ of single cubic $\mathrm{Cr}^{3+}$ centers in $\mathrm{MgO}: \mathrm{Cr}^{3+}(60 \mathrm{ppm})$ differs essentially from that for the 5.4-eV emission (see Fig. 8). The efficiency of $\mathrm{R}$-emission is high at $6.0-7.3 \mathrm{eV}$ in the region of the absorption of the oxygen ions located near $\mathrm{Cr}^{3+}$. The luminescence of single cubic $\mathrm{Cr}^{3+}$ centers contains also an inertial component caused by the recombination of holes with the $\mathrm{Cr}^{2+}$ centers formed at the electron trapping by $\mathrm{Cr}^{3+}$. This recombination emission is excited by the photons in the whole region of the fundamental absorption of $\mathrm{MgO}$. The excitation spectra of the $5.4 \mathrm{eV}$ emission and the $\mathrm{R}$-emission of $\mathrm{Cr}^{3+}$ are essentially different at $h v_{\mathrm{exc}}=$ $25-32 \mathrm{eV}$. According to the data of photoelectron spectroscopy, the excitation photons at these energies form hot conduction electrons able to create secondary e- $\mathrm{h}$ pairs. Because of high values of $E_{\mathrm{v}}$ and $E_{\mathrm{g}}$ in $\mathrm{MgO}$, there is a wide spectral region in the excitation spectra where the energy of hot carriers is insufficient for the creation of secondary $\mathrm{e}-\mathrm{h}$ pairs, whereas hot carriers with the energy excess of $4-6 \mathrm{eV}$ 
are able to participate efficiently at the direct excitation of $\mathrm{Cr}^{3+}$ centers. It should be stressed that even hot valence holes can excite the $\mathrm{R}$-emission of $\mathrm{Cr}^{3+}$ centers in $\mathrm{MgO}$. The efficient excitation of $\mathrm{Cr}^{3+}$ centers by hot centers is distinctive even in the crystals with relatively low concentration of impurities (60 ppm).

\section{Conclusion}

In conclusion, direct energy transfer by hot electrons or hot holes to luminescent impurity centers in WGCs $[10,30,35,45,46]$ can drastically decrease the efficiency of hot recombination of electrons with holes, thus serving as "luminescent defence" against the nonimpact mechanisms of radiation defect creation in the crystals with $E_{\mathrm{d}}>E_{\mathrm{g}}$. A further experimental and theoretical study of these processes lies ahead.

\section{Acknowledgements}

We are grateful to Prof. A. Shluger and Dr. P. Sushko for valuable discussions and to Drs. V. Babin, E. Feldbach, A. Kotlov, I. Kudryavtseva, T. Kärner, P. Liblik and A. Maaroos for assistance in some experiments. This work has been supported by the Estonian Science Foundation (grant 6652) and the European Community - Research Infrastructure Action under the FP6 "Structuring the European Research Area" Programme (through the Integrated Infrastructure Initiative "Integrating Activity on Synchrotron and Free Electron Laser Science").

\section{References}

[1] A.I. Ibarra, E.R. Hodson, Nucl. Instr. and Meth. B 218 (2004) 29.

[2] S.Y. Zinkle, C. Kinoshita, J. Nucl. Mater. 251 (1997) 200.

[3] Ch.B. Lushchik, I.K. Vitol, M.A. Elango, Sov. Phys. Usp. 20 (1977) 489.

[4] Ch.B. Lushchik, A.Ch. Lushchik, Decay of Excitonic Excitations with Defect Formation in Solids, Nauka, Moscow, 1989 (in Russian).

[5] K.S. Song, R.T. Williams, Self-Trapped Excitons, second ed., Springer, Berlin, Heidelberg, 1996.

[6] N. Itoh, A.M. Stoneham, Material Modification by Electronic Excitation, University Press, Cambridge, 2000.

[7] K. Matsunaga, T. Tanaka, T. Yamamoto, Y. Ikuhara, Phys. Rev. B 68 (2003) 085110.

[8] T. Brudevoll, E.A. Kotomin, N.E. Christensen, Phys. Rev. B 53 (1996) 7731.

[9] E. Feldbach, M. Kirm, A. Lushchik, Ch. Lushchik, I. Martinson, J. Phys.: Condens. Matter 12 (2000) 1991.

[10] M. Kirm, A. Lushchik, Ch. Lushchik, Phys. Status Solidi A 202 (2005) 213.

[11] L.X. Benedict, E.L. Shirley, R.B. Bohn, Phys. Rev. Lett. 80 (1998) 4514.

[12] N.-P. Wang, M. Rohlfing, P. Krüger, J. Pollmann, Phys. Rev. B 67 (2003) 115111.
[13] K.K. Rao, T.J. Moravec, J.C. Rife, R.N. Dexter, Phys. Rev. B 12 (1975) 5937

[14] R. Haensel, C. Kunz, B. Sonntag, Phys. Rev. Lett. 20 (1968) 262.

[15] E. Vasil'chenko, I. Kudryavtseva, A. Lushchik, Ch. Lushchik, V. Nagirnyi, Phys. Status Solidi C 2 (2005) 405.

[16] S. Nakonechnyi, T. Kärner, A. Lushchik, Ch. Lushchik, V. Babin, E. Feldbach, I. Kudryavtseva, P. Liblik, L. Pung, E. Vasil'chenko, J. Phys.: Condens. Matter 18 (2006) 379.

[17] A.A. Maiste, A.M.-E. Saar, M.A. Elango, JETP Lett. 18 (1973) 97.

[18] M.L. Dakss, R.L. Mieher, Phys. Rev. 187 (1969) 1053.

[19] Y.H. Chu, R.L. Mieher, Phys. Rev. Lett. 20 (1968) 1289.

[20] A. Lushchik, M. Kirm, Ch. Lushchik, Radiat. Meas. 33 (2001) 557.

[21] V.M. Agranovich, V.L. Ginzburg, Sov. Phys. JETP 13 (1961) 683.

[22] M. Kirm, A. Lushchik, Ch. Lushchik, I. Martinson, V. Nagirnyi, E. Vasil'chenko, J. Phys.: Condens. Matter 10 (1998) 3509.

[23] A. Lushchik, E. Feldbach, R. Kink, Ch. Lushchik, M. Kirm, I. Martinson, Phys. Rev. B 53 (1996) 5379.

[24] Yu.V. Kolk, A.Ch. Lushchik, Sov. Phys. Solid State 28 (1986) 805.

[25] A. Lushchik, M. Kirm, Ch. Lushchik, I. Martinson, F. Savikhin, Radiat. Eff. Defect. Solid. 155 (2001) 305.

[26] A. Lushchik, I. Kudryavtseva, Ch. Lushchik, E. Vasil'chenko, M. Kirm, I. Martinson, Phys. Rev. B 52 (1995) 10069.

[27] S. Guizard, P. Martin, Ph. Daguzan, G. Petite, Europhys. Lett. 29 (1997) 401.

[28] F. Quere, S. Guizard, Ph. Martin, G. Petite, H. Merdji, B. Carre, J.-F. Hergott, L. Le Deroff, Phys. Rev. B 61 (2000) 9883.

[29] D.I. Vaisburd, K.E. Evdokimov, Phys. Status Solidi C 2 (2005) 216.

[30] E. Feldbach, M. Kamada, M. Kirm, A. Lushchik, Ch. Lushchik, I. Martinson, Phys. Rev. B 56 (1997) 13908.

[31] D.I. Vaisburd, B.N. Semin, Bull. Russ. Acad. Sci., Phys. (USA) 56 (1992) 211

[32] K.U. Ibragimov, F.A. Savikhin, Phys. Solid State 35 (1993) 744.

[33] A. Lushchik, F. Savikhin, I. Tokbergenov, J. Lumin. 102-103C (2003) 44.

[34] F. Savikhin, M. Kerikmäe, E. Feldbach, A. Lushchik, D. Onishchik, D. Rakhimov, I. Tokbergenov, Phys. Status Solidi C 2 (2005) 252

[35] A. Lushchik, Ch. Lushchik, E. Feldbach, I. Kudrjavtseva, P. Liblik, A. Maaroos, V. Nagirnyi, E. Vasil'chenko, F. Savikhin, Proc. SPIE 5946 (2005) 594609.

[36] Y.N. Xu, W.Y. Ching, Phys. Rev. B 43 (1991) 4461.

[37] I.V. Kityk, B.V. Andrievskii, V.O. Yuvshenko, Phys. Status Solidi B 182 (1994) K79.

[38] E. Calabrese, W.B. Fowler, Phys. Rev. B 18 (1978) 2888.

[39] Y.P. Li, W.Y. Ching, Phys. Rev. B 31 (1985) 2172.

[40] A.L. Shluger, P.V. Sushko, Private communication.

[41] A. Trukhin, P. Liblik, Ch. Lushchik, J. Jansons, J. Lumin. 109 (2004) 103.

[42] M. Cannas, S. Agnello, F.M. Gelardi, R. Boscaino, A. Trukhin, P. Liblik, C. Lushchik, M. Kink, Y. Maksimov, R. Kink, J. Phys.: Condens. Matter 16 (2004) 7931.

[43] M. Kirm, G. Zimmerer, E. Feldbach, A. Lushchik, Ch. Lushchik, F. Savikhin, Phys. Rev. B 60 (1999) 502.

[44] A. Lushchik, M. Kirm, Ch. Lushchik, I. Martinson, V. Nagirnyi, E. Vasil'chenko, Nucl. Instr. and Meth. B 191 (2002) 135.

[45] A. Lushchik, M. Kamada, M. Kirm, Ch. Lushchik, I. Martinson, Radiat. Meas. 29 (1998) 229.

[46] M. Kirm, E. Feldbach, T. Kärner, A. Lushchik, Ch. Lushchik, A. Maaroos, V. Nagirnyi, I. Martinson, Nucl. Instr. and Meth. B 141 (1998) 431. 